\title{
Influence of in vitro growth conditions in the production of defence compounds in Mentha pulegium L.
}

\author{
Khadija H. Benlarbi a , Nouriddine Elmtili ${ }^{\text {b }}$, Francisco A. Macías ${ }^{\text {a,* }}$, Juan Carlos G. Galindo ${ }^{a}$ \\ ${ }^{a}$ Laboratory of Biology and Health, Department of Biology, Team of Biotechnology, Faculty of Science, Abdelmalek Essaadi University, BP 2121093002, \\ Tetouan, Morocco \\ ${ }^{\mathrm{b}}$ Allelopathy Group, Department of Organic Chemistry, Campus de Excelencia Internacional Agroalimentario (ceiA3), Faculty of Sciences, University of Cádiz, \\ C/ República Saharaui, s/n, 11510 Puerto Real, Cádiz, Spain
}

\section{A R T I C L E I N F O}

\section{Article history:}

Received 29 January 2014

Received in revised form 4 March 2014

Accepted 12 March 2014

Available online 4 April 2014

\section{Keywords:}

Mentha pulegium

Pulegone

Micropropagation

Allelopathy

Plant defence

Abiotic stress

\begin{abstract}
A B S T R A C T
The content in secondary metabolites greatly affects the quality of plants used for food consumption. Specifically, the production of defence secondary metabolites is triggered by a number of biotic and abiotic stress factors. Commonly studied biotic factors include insects and other herbivores that cause wounding and tissue damage due to microorganisms. Among abiotic factors, mechanical stress induced in the roots has yet to be studied in depth. Such studies are of interest because of the influence of this factor on plants grown under in vitro conditions. Herein, we present our results on essential oil production and other defence-related morphological traits in terms of the effect of mechanical stress induced in the roots of Mentha pulegium plants grown under different conditions: greenhouse using soil as support and non-soil in vitro conditions using gelose and glass beads. A positive correlation has been found between secondary metabolites production, i.e. essential oil and growth conditions. The presence and development of morphological characteristics linked to their storage and lixiviation into the environment - trichomes and glandular hairs in the leaves; lateral roots and fibrous roots - has also been positively related to an increase in penetration resistance on the roots by the substrate - mechanical impedance. Pulegone is the major defence compound in pennyroyal essential oil. Pulegone production and other essential oil constituents present in trichomes have been quantified under different growth conditions and the results have been correlated with morphological characteristics and growth conditions.
\end{abstract}

(c) 2014 Phytochemical Society of Europe. Published by Elsevier B.V. All rights reserved.

\section{Introduction}

Throughout the course of their growth and development, plants are exposed to a variety of environmental stress factors - both biotic and abiotic - that have an enormous influence on plant primary and secondary metabolic production. Abiotic stress factors such as cold (Maruyama et al., 2009), drought (Andre et al., 2009), UV radiation (Conconi et al., 1996), heavy metals (Tumova et al., 2007) and high salinity (Nasir Khan et al., 2010) have a strong influence on gene expression and have been previously studied in many crops. However, mechanical stresses other than wounding or tissue damage due to atmospheric or pathogen attack have not been studied extensively (Bengoung et al., 2011). Mechanical impedance - defined as penetration resistance on the roots by the soil or the substrate - is present at the roots from the very

\footnotetext{
* Corresponding author. Tel.: +34956 016405; fax: +34 956016193.

E-mail address: famacias@uca.es (F.A. Macías).
}

beginning of plant growth, and the cellular responses of roots fundamentally influence the development of secondary morphological characteristics such as morphogenesis, root hair development, root length and diameter (Braam, 2005; Okamoto et al., 2008). Moreover, mechanical impedance is a factor to be considered when it comes to grown plants under hydroponics or non-soil in vitro culture conditions.

To our knowledge, the possible connection between mechanical impedance and the development of other secondary defence organs, i.e. glandular trichomes and leaf hairs, has not been documented to date. The morphology and phenotypic expression of roots during mechanical impedance has been shown to be strikingly similar to that observed in ethylene-treated seedlings in a number of species (Kays et al., 1975; Goeschl et al., 1996) thus suggesting that signal transduction to the rest of the plant due to hormonal production and release may be involved in the development of secondary organs in parts of the plant other than the roots. Some of these organs are of importance in plant defence (Duffey, 1986) and secondary metabolite production (Eisner et al., 
1990; Wagner, 1991). Therefore, the large scale production of higheconomic-value compounds through callus and in vitro culture requires an in depth understanding of the influence that such factors have on metabolite production.

Lamiaceae families are known to produce a wide array of secondary metabolites and are a rich source of economically valuable terpenes. Among them, Mentha pulegium L. (pennyroyal) is characterized by a high content of essential oils ( $4 \%$ of the dry weight), the main component of which is the bioactive monoterpene ketone pulegone (Thomassen et al., 1990; Aghel et al., 2004; Sampson et al., 2005; Diaz-Maroto et al., 2007). Pulegone has been related with defence roles against herbivory in wild thyme (Müller-Schwarze and Thoss, 2008) and has shown insecticidal (Liu et al., 2011; Sampson et al., 2005) antibacterial (e.g., Teixeira et al., 2013) activities, among others. Also, an increase in pulegone production in Minthostachys mollis has been observed as a consequence of leaf damage - leaf puncturing - simulating the effect of leaf miners (Banchio et al., 2007).

Pennyroyal is a well-known plant that is widely used in folk medicine in many cultures for aromatherapy, abortifacient and as a remedy for colic. The oil is also commercially used as a flavouring agent in beverages and foods. However, the essential oil has been associated also with hepatotoxicity. Cytochrome P 450-mediated oxidation of pulegone generates menthofuran (McClanahan et al., 1989) which can be further metabolized into reactive compounds that can form adducts to hepatocellular proteins (Khojasteh et al., 2012). Therefore, the interest of studying the factors affecting pulegone and essential oil production in pennyroyal and why this species was selected as a model system to study the physiological changes and variation in secondary metabolite production induced by abiotic environmental factors.

In addition to phenotype selection, an understanding of the interactions between secondary metabolic pathways and the environment is a prerequisite for the optimization of secondary metabolite production (Sharma et al., 2002). Metabolic engineering has been suggested as a promising technology for the production of phytochemicals (Croteau et al., 2000), and knowledge of the factors that influence the distribution and accumulation of secondary metabolites in plant tissue and organs is imperative. In this sense, high frequency tissue culture systems and efficient culturing systems aimed at expressing the target phytochemical(s) are attractive options for the mass production of high-value secondary metabolites (Canter et al., 2005). Tissue culture using micropropagation has been suggested as a promising technology for physiological and phytochemical studies (Murch et al., 2000); this method can also play an important role in clonal propagation of high quality plants (Sahoo and Chand, 1998; Rathore et al., 2004; Liu et al., 2006, 2008) and in the mass production of secondary metabolites (Prakash et al., 2002; Yoshimatsu, 2008). The use of explants cultures also ensures higher genetic stability in comparison with callus cultures, thus allowing a better standardization of secondary metabolic production.

The present study with M. pulegium L. offers some clues to understanding the physiological and phytochemical state of plants affected by culture conditions and environmental factors such as mechanical impedance (expressed through changes in the support matrix) and water levels. Knowledge about the responses of plant morphogenesis and secondary metabolic production to the culturing parameters is needed to provide potential targets for metabolic engineering. In this study we specifically investigated the effects of two culture conditions (oil and non-soil) using three different supports (agar, glass beads and soil) on: (1) biomass production and relative growth rate of shoots and roots; (2) chemical status: essential oil (pulegone) production and essential oil profiling; and (3) histochemical status of storage glands (trichomes and leaf hair density) and root hairs.
The aims of the experiments presented here were as follows: (a) to establish a correlation between the chemical (essential oil profile and production), the histological and physiological status, and (b) to determine whether growth conditions would influence the production of the allelochemical pulegone and other essential oil components.

\section{Results and discussion}

Micro-shoots retain morphogenetic potential over the generations and can be further multiplied or rooted. Alternatively, their potential for aseptic biomass production and mass production of secondary metabolites can also be explored and, eventually, exploited, and this is one of the main purposes of this study. Accordingly, the effects of growth conditions (with a special attention paid to mechanical impedance) on morphogenesis, chemical profile and secondary metabolite production will be discussed. In the present study, the in vitro shoot segments were used as a source of explants for the production of multiple shoots through the de novo shoot regeneration because cutting simulates the effect of wounding and is known to promote re-differentiation in explants under in vitro conditions (Vinocur et al., 2000).

\subsection{Morphogenesis in vitro (non-soil conditions)}

Plant growth and developmental responses of micro-propagated shoots to different culture and environmental conditions (solidified agar, glass bead support, and acclimatized plants grown in pots) characterized by different degrees of mechanical impedance are shown in Tables 1 and 2. Agar medium was selected as reference or control for the minimum level of mechanical impedance. The results in Table 1 clearly show enhanced growth through an increase in biomass production and growth index (GI) in plants grown on glass beads in comparison with those grown in agar conditions. This increment is also correlated with a higher number of lateral roots (Fig. 1) and continuous production of lateral roots and shoots on plants grown under the two conditions (agar and glass beads) was observed. Improved nutrient and oxygen uptake enhanced biomass growth because tissue was supported on a floating raft that kept the tissue in constant contact with the medium. However, the most marked differences were observed in the number of lateral roots: plants grown on glass beads showed a fourfold increase with respect to those grown in agar, a finding that can be explained as being a direct result of the higher mechanical impedance caused by the glass beads (Fig. 1). These data are indicative of the importance of mechanical impedance in the development of secondary morphological characteristics such as lateral roots. Whether this effect could be transferred to parts of the plant other than the roots is an issue that has not been proven yet.

Table 1

Caulogenese response of in vitro micro-propagated shoots of Mentha pulegium L. after 30 days of subculture under two different sets of conditions in terms of mechanical impedance: solid (agar) and glass bead support. ${ }^{a}$

\begin{tabular}{lcl}
\hline & Agar medium & Glass bead \\
\hline Biomass (g) & $9.20 \pm 0.006^{\mathrm{a}}$ & $10.86 \pm 0.006^{\mathrm{b}}$ \\
Growth index (GI) & $21.43 \pm 0.005^{\mathrm{a}}$ & $25.49 \pm 0.005^{\mathrm{b}}$ \\
No. of shoots & $16.10 \pm 0.01^{\mathrm{a}}$ & $20.05 \pm 0.01^{\mathrm{b}}$ \\
Shoot lengths & $12.000 \pm 0.007^{\mathrm{a}}$ & $14.25 \pm 0.09^{\mathrm{b}}$ \\
No of roots & $15.12 \pm 0.006^{\mathrm{a}}$ & $17.31 \pm 0.006^{\mathrm{b}}$ \\
No. of lateral roots & $4.32 \pm 0.006^{\mathrm{a}}$ & $17.15 \pm 0.06^{\mathrm{b}}$ \\
\hline
\end{tabular}

Values correspond to the mean of 10 measurements \pm standard error. Values within the same line with different letters $(\mathrm{a}, \mathrm{b}$, or $\mathrm{c}$ ) are different at $P<0.01$.

a Values significantly different with $P<0.01$.

b Values significantly different with $0.01<P<0.05$. 
Table 2

Physiological and biochemical status of in vitro and in vivo plantlets.

\begin{tabular}{|c|c|c|c|}
\hline & Solid agar (in vitro) & Glass beads (in vitro) & Plantlets in pots (in vivo) \\
\hline RGR (g day ${ }^{-1}$ plant $^{-1}$ ) & $0.06 \pm 0.001^{\mathrm{a}}$ & $0.08 \pm 0.004^{\mathrm{a}}$ & $0.02 \pm 0.002^{\mathrm{b}}$ \\
\hline $\mathrm{Gnd}^{\mathrm{b}}$ & $0.30 \pm 0.01^{\mathrm{a}}$ & $0.36 \pm 0.002^{\mathrm{b}}$ & $0.14 \pm 0.01 \mathrm{c}$ \\
\hline Leaf area $\left(\mathrm{cm}^{-2}\right)$ & $3.17 \pm 0.01^{\mathrm{a}}$ & $3.24 \pm 0.012^{\mathrm{b}}$ & $2.81 \pm 0.01 c$ \\
\hline LWC (\%) & $81.45 \pm 0.02^{\mathrm{a}}$ & $85.19 \pm 0.04^{\mathrm{b}}$ & $60.80 \pm 0.05 c$ \\
\hline Total chlorophyll ( $\mu \mathrm{g} / \mathrm{mg}$ fresh leaf) & $3.23 \pm 0.005^{\mathrm{a}}$ & $3.96 \pm 0.004^{b}$ & $2.26 \pm 0.006 c$ \\
\hline Chlorophyll $a$ & $2.02 \pm 0.006^{\mathrm{a}}$ & $2.45 \pm 0.004^{b}$ & $1.35 \pm 0.004 c$ \\
\hline Chlorophyll $b$ & $1.21 \pm 0.006^{\mathrm{a}}$ & $1.51 \pm 0.002^{\mathrm{b}}$ & $0.91 \pm 0.004 \mathrm{c}$ \\
\hline Glandular trichome (number $\mathrm{mm}^{-2}$ ) & $23.2 \pm 1.72^{\mathrm{a}}$ & $24.5 \pm 2.05^{\mathrm{a}}$ & $56.3 \pm 1.82^{\mathrm{b}}$ \\
\hline Leaf hairs (number $\mathrm{mm}^{-2}$ ) & $8.3 \pm 1.64^{\mathrm{a}}$ & $21.9 \pm 2.21^{\mathrm{b}}$ & $125.4 \pm 3.28 c$ \\
\hline Volatiles ( $\mathrm{mg} \mathrm{g}^{-1}$ fresh tissue) ${ }^{\dagger}$ & 0.026 & 0.028 & 0.241 \\
\hline
\end{tabular}

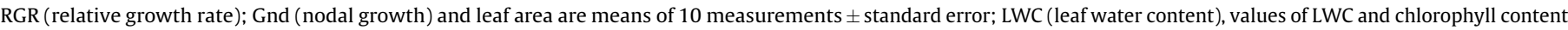
are means of 50 leaves \pm standard error. Values within the same line with different letters $(\mathrm{a}, \mathrm{b}$, or $\mathrm{c}$ ) are different at $P<0.01$.

There are no statistics for volatiles (essential oils) content as only one extraction (50 leaves) was performed per growth condition.

a Values significantly different with $P<0.01$

b Values significantly different with $0.01<P<0.05$.

Significant differences in relative leaf water content (LWC) were also observed between plants grown under in vitro and soil conditions, but not between the two in vitro culture conditions (Table 2). Plants growing under soil conditions present a lower LWC. There is also a small but statistically significant difference in the LWC between plants grown in agar and glass beads. A similar pattern can be observed in the chlorophyll content (Table 2, entries 4-6). Reduction in chlorophyll contents have been correlated previously with water stress (Pirzad et al., 2011). However, whether this difference in LWC for in vitro and soil conditions can be partly attributed to a certain degree of water stress is unclear.

The higher number of lateral roots observed in the glass beads culture can also be correlated with the increase in the GI because the cut surfaces are better exposed to the medium. The liquid culture - while beneficial for bioreactor scale-up and plant growth - often induces hyper hydration (Aitken-Christie et al., 1995). This phenomenon is often attributed to the presence of a liquid layer on plants grown in the liquid medium, which in turn results in poor gas exchange (Etienne and Berthouly, 2002). Our results show that micro-propagated plants produced more fresh and dry weight in liquid/glass beads than in a solid medium. This finding suggests that an increased uptake of water and nutrients occurs in the presence of liquid media (glass beads) when the plants have a normal morphology. This liquid/glass matrix was also recommended in other in vitro studies on the basis of whole plant physiology (Kenneth and Jerzy, 1990) and has also been successfully used as a support matrix for mass production and cost efficient propagation (Goel et al., 2007).

\subsection{Physiological and histochemical study}

In all cases, plants grown under in vitro conditions grew more vigorously and exhibited better growth bio-kinetics than those plants raised in pots in a greenhouse (Table 2), as shown by the values for Relative Growth Rate (RGR), nodal growth (Gnd) and leaf area. In accordance with the caulogenese - induction of shoot development from callus - response mentioned above, plants grown on glass beads showed a slight - but significant - increase in their growth parameters.

Glandular trichomes are reservoirs of essential oils and have been related with defensive roles as chemical weapons arsenals (Gang et al., 2002). The number of glandular (peltate) trichomes is not affected by the mechanical impedance exerted on roots in the change from the agar to glass bead support (Table 2, entries 8 and 9 ), but this number is markedly higher in plants grown in pots under greenhouse conditions. In all cases the number of glandular trichomes presented a random distribution on both abaxial and adaxial sides of the leaves. Similar behaviour is also observed for the total amount of volatiles obtained by ultrasound treatment (Fig. 2). The number of leaf hairs seems to be even more sensitive to culture conditions: significant differences were observed between agar and glass bead conditions and also between these plants and those grown in pots and raised in the greenhouse. Furthermore, the degree of development of leaf hairs appears to be higher in plants grown in the greenhouse than those grown under in vitro conditions (Fig. 3).

It is well known that stress conditions cause an increase in the production of defence secondary metabolites. The dilemma of defence vs. growth is that the synthesis of these compounds involves a flow of resources (carbon, enzymes and energy-rich molecules such as ATP or NADPH) from primary to secondary metabolism and this is directly related with defence or growth/ development costs (Herms and Mattson, 1992). It was previously mentioned that a lower LWC was observed in greenhouse plants, but we do not have data supporting a correlation between these levels and a certain water stress besides the fact that chlorophyll contents were also lower. However, the mechanical impedance exerted by the soil on the roots is by far higher than that in plants
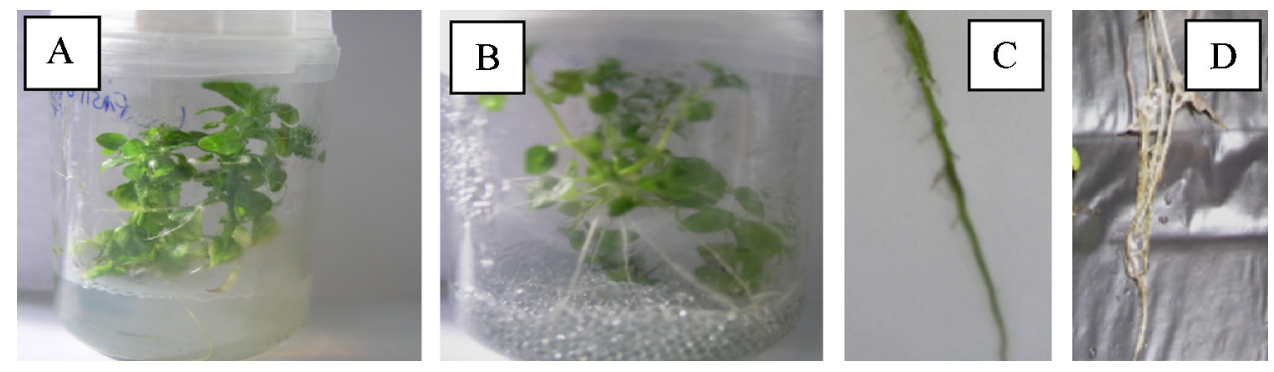

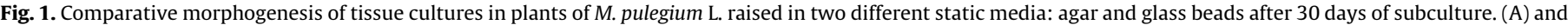

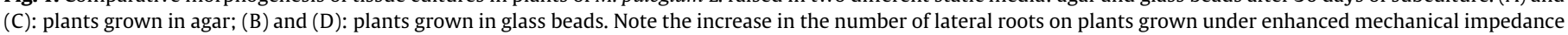
(right, glass beads). 


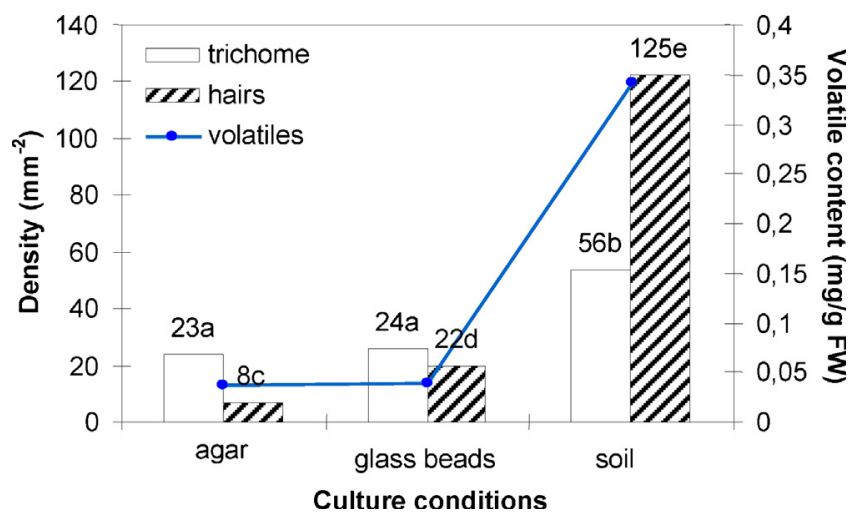

Fig. 2. Density (number $\mathrm{mm}^{-2}$ ) of trichomes and leaf hairs of $M$. pulegium L. plants raised under different growth conditions: agar, glass beads and pots. Note the parallel increase of leaf glandular organs (trichomes and hairs) and volatiles in plants grown in soil in the greenhouse (in vivo). Values with different letters in each series (trichomes or hairs) are statistically different at 0.95 (Student's $t$ test).

grown on glass beads. And so it happens with light intensity in the greenhouse as compared to in vitro conditions. These facts correlate well with the tenfold increase observed in the amount of defence volatiles produced in the trichomes of greenhouse plants in comparison with plants grown in vitro. Moreover, the number of leaf hairs increased with mechanical impedance, as did the number of glandular trichomes containing essential oils.

Finally, the external appearance of peltate trichomes is also different depending on the growth conditions, as shown in the SEM micrographs: glandular trichomes of plants grown in agar solidified and over glass beads have an "empty" appearance (Figs. 3 and 4), similar to that of trichomes of greenhouse plants after selective essential oil extraction; meanwhile, trichomes of greenhouse plants have a "full" external appearance that is turgid and swollen (Fig. 4). This observation correlates well with the different essential oil contents shown in Table 2, where a steady decrease in the amount of volatiles produced can be seen depending on the growth conditions (greenhouse, hydroponics in glass beads and agar). The analysis by families of compounds shows also a similar pattern for monoterpene and methyl esters of fatty acid contents. This behaviour is not as clear in the volatile sesquiterpene fraction.

The question arises as to whether there is any connection between root response to mechanical impedance and the development of certain morphologic characteristics in other parts of the plant. The mechanical impedance is generally assumed to be perceived in the cells of the root tips through a change in the membrane voltage and this is transmitted via calcium channels
(Fasano et al., 2002). This process can induce expression of some genes in shoots (Sistrunk et al., 1994; Xu et al., 1995), thus connecting this stimulus with responses in other parts of the plant. Despite the fact that there is still debate as to whether ethylene synthesis or ethylene response is the main factor that regulates root growth and morphology during mechanical impedance, the latest data suggest that an altered ethylene response regulates the growth and morphology of roots during mechanical impedance (Okamoto et al., 2008) and this implies a hormonal response to this stress factor. As a consequence, the development of secondary characteristics in parts of the plants other than those directly in contact with the source of the mechanical impedance is a convenient working hypothesis. The way in which signal transduction takes place is still far from understood, but the available data point to a hormonal response since the expression of touch-inducible $(\mathrm{TCH})$ genes can be induced by a variety of hormones (Sistrunk et al., 1994).

\subsection{Production of volatile compounds}

Defence volatiles are stored mainly in glandular trichomes and not in other organs. All trichomes develop very early in the life of the leaf. Trichomes are formed from a protodermal cell, prior to division, and this process requires reorganization and nuclear migration to the upper (apical) end of the cell. This is followed by the production of large vacuoles at the bottom of the cell. The cell divides asymmetrically and this leads to the formation the trichome (Karousou et al., 1992; Ascensão et al., 1995). The expression of certain secondary metabolites is most likely associated with organ differentiation and not biomass accumulation (Herms, 1999). Consequently, the stage of development of these organs - and those environmental factors that affect it - will greatly influence the volatile content. According to this hypothesis, the higher number of developed defence organs (i.e. glandular trichomes and leaf hairs) and the increase in the production of defence volatiles - as shown by the total production and the appearance and developmental state of the glandular trichomes and leaf hairs obtained from the SEM study - could be correlated with the change from in vitro (agar or glass beads) to greenhouse conditions. The effect of increased mechanical stress induced in the roots first appears on the production of lateral roots from agar to glass beads. However, the full expression of secondary organs takes place in the change to plants grown in pots under greenhouse conditions. There are many environmental factors that are drastically modified in this last transition - light intensity, possible water stress, and mechanical impedance. Among them the increased mechanical impedance should not be rejected as of influence as water stress was tried to keep at minimum with the plants being regularly watered in the greenhouse. However light

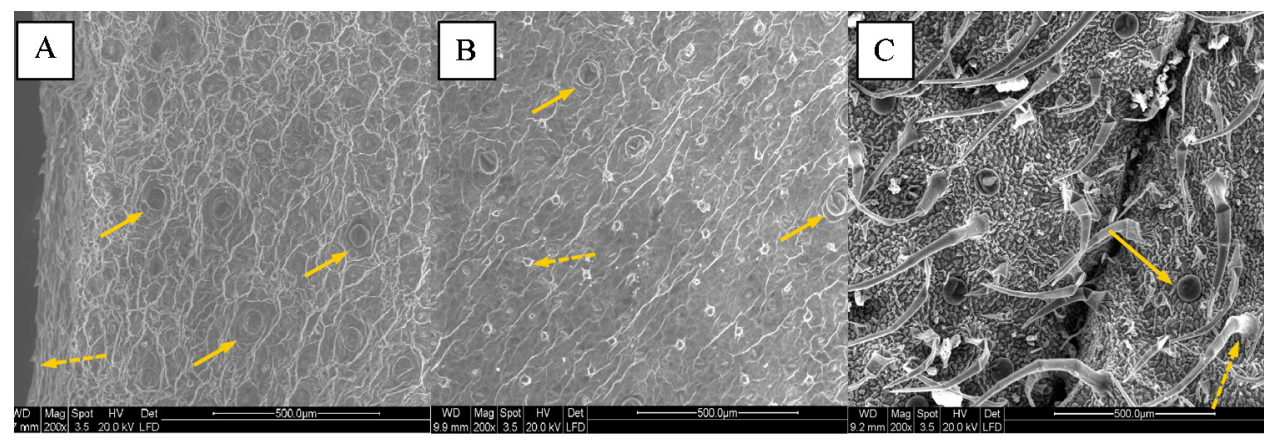

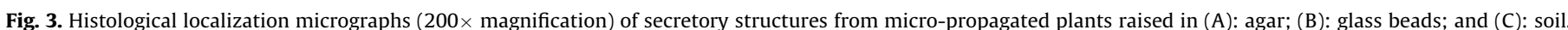

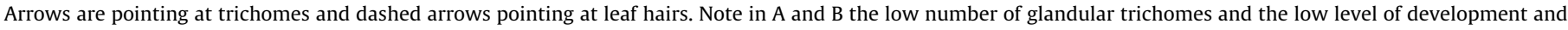
number of leaf hairs in comparison to $\mathrm{C}$. 


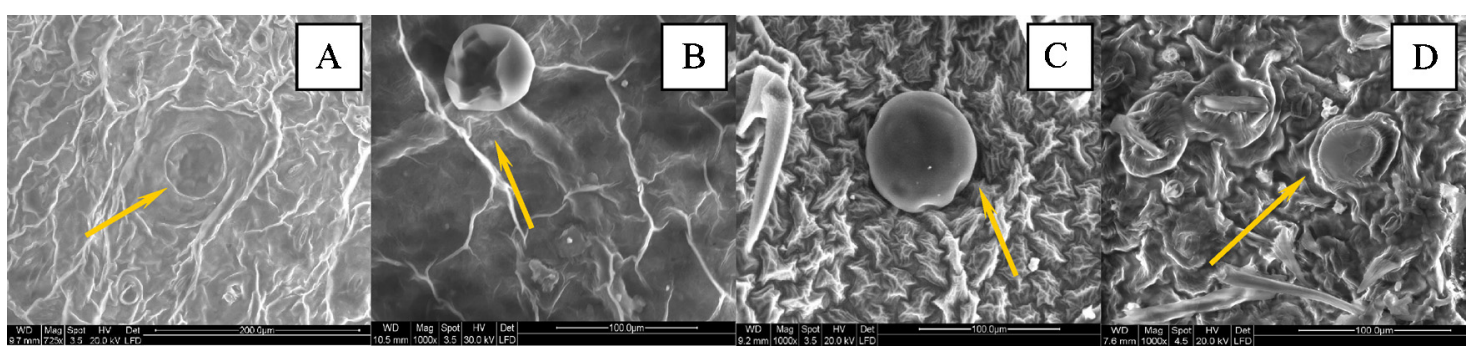

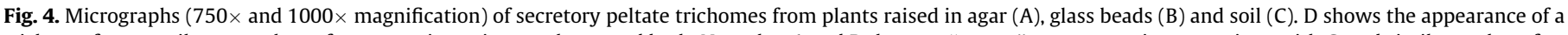

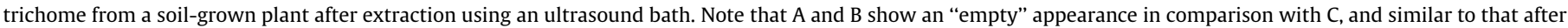
extraction (D).

intensity also increased in the greenhouse and should be considered as another key factor. We propose that the effect of mechanical impedance would add to the overall environmental effect and could be transmitted via hormones to the rest of the plant.

In this study we only compared volatile compounds contained in the trichomes and obtained from the de novo apical leaves during the vegetative stage and after complete development in the case of in vitro plants. Despite the fact that there was an increase in biomass on changing from solidified media (agar) to liquid media (glass beads) in plants grown under in vitro conditions, this was not accompanied by any significant increase in the production of volatile compounds (Table 1; Fig. 2). This suggests that in the absence of strong stress conditions, a high degree of morphogenesis (biomass, leaf surface) during in vitro culture led to the preferential flow of carbon and energy towards growth and primary metabolism. It appears that the increase in the mechanical impedance from agar to glass beads is not enough to trigger massive essential oil production. This finding is in good agreement with the theories in which it is proposed that a balance must exist between carbon allocation for growth and for the synthesis of defence compounds (Kenneth and Jerzy, 1990).

The results of GC-MS analyses are shown in Tables 3 and 4. Compounds were identified by comparison of their retention times with those of standards and/or with their mass spectra (NIST'98 library). A total of 18 monoterpenes, 6 sesquiterpenes, a long chain alcohol (phytol) and 11 methylated fatty acids (MEFA) were identified and quantified in the essential oil contained in the trichomes of plants grown under the three different growth conditions studied. However, striking differences were found

Table 3

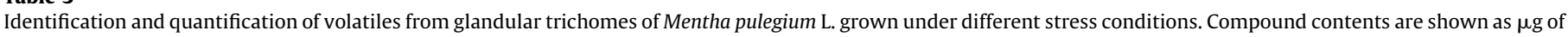
compound per gram of FW (fresh weight) leaves.

\begin{tabular}{|c|c|c|c|c|c|c|c|c|}
\hline & \multirow[t]{3}{*}{ MW } & \multirow[b]{2}{*}{ r.t. } & \multicolumn{2}{|l|}{ Soil } & \multicolumn{2}{|c|}{ Glass beads } & \multicolumn{2}{|l|}{ Agar } \\
\hline & & & RSI & $\mu \mathrm{g} / \mathrm{mg} \mathrm{FW}$ & RSI & $\mu \mathrm{g} / \mathrm{mg} \mathrm{FW}$ & RSI & $\mu \mathrm{g} / \mathrm{mg}$ FW \\
\hline & & \multicolumn{7}{|c|}{ Monoterpenes } \\
\hline 1,8-Cineole & & 9.62 & n.d. & & n.d. & & 864 & $10.27 \pm 0.92$ \\
\hline Camphene & & 10.28 & 877 & $92.85 \pm 5.02$ & 788 & $0.66 \pm 0.13$ & n.d. & \\
\hline Unid. (many possible comp.) & & 10.56 & & $35.56 \pm 2.32$ & n.d. & & n.d. & \\
\hline p-Menth-8-en-3-ol (isopulegol) ${ }^{a}$ & & 11.55 & 784 & $15.38 \pm 3.76$ & n.d. & & n.d. & \\
\hline Menthone (dihydropulegone) & & 11.81 & n.d. & & n.d. & & 965 & $24.67 \pm 0.28$ \\
\hline$p$-Menth-1-en-8-ol & & 11.84 & n.d. & & n.d. & & & $12.68 \pm 0.33$ \\
\hline$p$-Menth-8-en-3-one (isopulegone) or cyclocitral/pinocamphone ${ }^{\mathrm{c}}$ & & 11.99 & 836 & $5.13 \pm 0.57$ & n. d. & & 909 & $10.80 \pm 0.44$ \\
\hline Terpinen-4-ol & & 12.21 & n.d. & & n.d. & & 919 & $17.38 \pm 0.46$ \\
\hline Terpineol & 154 & 12.56 & 807 & $43.09 \pm 3.30$ & n.d. & & n.d. & \\
\hline Pulegone $^{a}$ & & 12.94 & 911 & $1357.35 \pm 73.49$ & 912 & $110.50 \pm 8.24$ & 887 & $5.59 \pm 0.00$ \\
\hline Isopulegyl acetate & 196 & 13.56 & 882 & $4.69 \pm 0.19$ & n.d. & & n.d. & \\
\hline p-Menth-8-en-2-ol acetate & 196 & 13.64 & 840 & $71.04 \pm 7.98$ & n.d." & $8.58 \pm 0.63$ & n.d. & \\
\hline Unidentified & & 13.86 & n.d. & & n.d. & & & $1.27 \pm 0.13$ \\
\hline 3,7,11-Trimethyl-2,6,20-dodecatrienal & 220 & 13.90 & 685 & $17.26 \pm 2.11$ & n.d. & & n.d. & \\
\hline$\gamma$-Terpineol & 154 & 14.01 & 803 & $509.45 \pm 46.40$ & 767 & & n.d. & \\
\hline Carveyl acetate & 194 & 14.21 & 834 & $40.35 \pm 3.34$ & n.d. & & n.d. & \\
\hline Elemene & & 14.37 & n.d. & & n.d. & & 829 & \\
\hline Chrysanthenone $^{\mathrm{b}}$ & 150 & 14.39 & 872 & $87.16 \pm 6.68$ & 834 & $2.12 \pm 0.13$ & n.d. & \\
\hline$\beta$-Caryophyllene & 204 & 15.52 & 875 & $12.33 \pm 0.94$ & n.d. & & 928 & $18.71 \pm 0.64$ \\
\hline Humulene & 204 & 15.96 & 877 & $13.78 \pm 0.71$ & n.d. & & 819 & $1.69 \pm 0.02$ \\
\hline Germacrene D & 204 & 16.30 & n.d. & & n.d. & & 993 & $6.78 \pm 0.15$ \\
\hline Germacrene B & 204 & 16.49 & n.d. & & n.d. & & 926 & $3.95 \pm 0.63$ \\
\hline Viridiflorol & 204 & 17.39 & n.d. & & n.d. & & 832 & $2.00 \pm 0.04$ \\
\hline Spathulenol & 220 & 17.49 & n.d. & & n.d. & & 851 & $5.26 \pm 0.17$ \\
\hline Phytol & 296 & 26.81 & 718 & $10.05 \pm 3.01$ & n.d. & & n.d. & \\
\hline Total monoterpenes & & & & 2262.09 & & 121.88 & & 80.92 \\
\hline Total sesquiterpenes & & & & 43.36 & & 0.0000 & & 31.13 \\
\hline Total terpenoids & & & & 2315.50 & & 121.88 & & 112.05 \\
\hline
\end{tabular}

a Confirmed by r.t. of a standard; Pulegone conc. was calculated using a calibration curve with a commercial pure sample (regression for the areas: $y=165,484+1.77804 \times 10^{6} x ; r^{2}=0.9968$ FID); RSI: confidence value given by the library (NIST).

b Not previously reported in M. pulegium; tentative identification based on library (NIST'98) search.

c could be either cyclocitral or p-meth-8-en-3-one in the agar sample; not detected in glass beads; identified as pinocamphone in the green-house sample.

The mass spectrum is similar to that of the identified compound in sample 1, but the peak is too low and a peak at 153.1 does not match the identification. 
Table 4

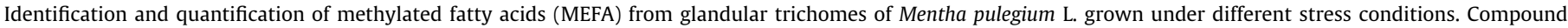
content is shown as $\mu \mathrm{g}$ of compound per gram of FW leaves.

\begin{tabular}{|c|c|c|c|c|c|c|c|}
\hline & \multirow[b]{2}{*}{$\begin{array}{l}\text { r.t. } \\
\text { Fatty acids }\end{array}$} & \multicolumn{2}{|l|}{ Soil } & \multicolumn{2}{|c|}{ Glass beads } & \multicolumn{2}{|l|}{ Agar } \\
\hline & & RSI & $\mu \mathrm{g} / \mathrm{mg}$ FW & RSI & $\mu \mathrm{g} / \mathrm{mg}$ FW & RSI & $\mu \mathrm{g} / \mathrm{mg}$ FW \\
\hline Caprylic acid methyl ester ${ }^{\mathrm{a}}$ & 11.14 & 864 & $28.34 \pm 4.49$ & n.d. & & n.d. & \\
\hline Decanoic acid methyl ester ${ }^{\mathrm{a}}$ & 14.07 & 899 & $210.9 \pm 14.27$ & n.d. & & n.d. & \\
\hline Methyl palmitoleate $^{\mathrm{a}}$ & 20.90 & 904 & $188.01 \pm 41.86$ & Trace & $0.82 \pm 0.06$ & n.d. & \\
\hline Methyl palmitate $^{a}$ & 21.10 & & $690.85 \pm 117.38$ & & & & \\
\hline Methyl linolenate $^{a}$ & 22.74 & 897 & $118.25 \pm 37.27$ & Trace & $0.88 \pm 0.17$ & Trace & \\
\hline Methyl oleate $^{\mathrm{a}}$ & 22.80 & 859 & $453.90 \pm 138.61$ & Trace & $0.74 \pm 0.09$ & Trace & \\
\hline Methyl stearate ${ }^{a}$ & 23.02 & 924 & $387.06 \pm 110.39$ & Trace & $0.82 \pm 0.27$ & Trace & \\
\hline Methyl arachidate $^{a}$ & 24.78 & 878 & $252-62 \pm 85.43$ & n.d. & & n.d. & \\
\hline Methyl eruciate ${ }^{a}$ & 26.22 & 826 & $59.56 \pm 13.62$ & n.d. & & n.d. & \\
\hline Methyl behenate ${ }^{\mathrm{a}}$ & 26.40 & 860 & $155.70 \pm 41.30$ & 606 & & n.d. & \\
\hline Methyl lignocerate ${ }^{a}$ & 27.91 & & $79.21 \pm 12.80$ & & & & \\
\hline Total MEFA & & & 2634.80 & & 3.26 & & 0.0000 \\
\hline
\end{tabular}

a Confirmed by r.t. of a standard; trace: same r.t. but not identified by mass spectrum.

when compounds were analyzed by families and growth conditions.

Table 5 enables a comparison of the compounds reported in the bibliography as components of pennyroyal essential oil obtained by hydrodistillation (Diaz-Maroto et al., 2007; Karray-Bouraoui et al., 2009) or supercritical liquid extraction (Aghel et al., 2004) with those obtained in the present study by selective ultrasonic extraction. Only the monoterpenes $\alpha$-pinene, $\beta$-pinene, 1,8cineole, 4-terpinyl acetate, camphene, isopulegol, pulegone, piperitenone, terpinen-4-ol and viridiflor, the sesquiterpenes $\alpha$ humulene, $\alpha$-phellandrene, $\beta$-caryophyllene, germacrene $D$, and the fatty acids methyl linoleate, methyl stearate and methyl palmitate appear in the essential oils obtained using the different extraction techniques. Moreover, the methyl esters of caprylic, decanoic, palmitoleic, oleic, arachidic, erucic, behenic and lignoceric fatty acids were detected in the ultrasonic extracts and these were not previously reported for the other techniques. A further 43 compounds reported in the literature could not be detected in the essential oil obtained using ultrasound extraction.

The results show that this methodology leads to the selective extraction of those compounds present in the leaf storage glands (peltate and other glandular trichomes) and not of those in the inner tissues. These compounds are more likely to be released into the environment than those stored in the cells deep within the leaf.

Table 5

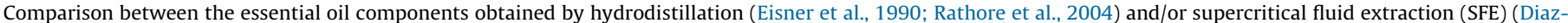

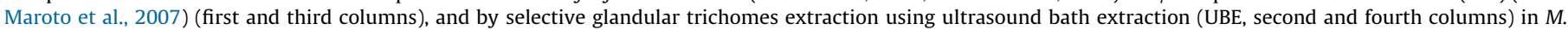
pulegium L.

\begin{tabular}{|c|c|c|c|}
\hline Compound & UBE detection $^{\mathrm{a}}$ & Compound & UBE detection $^{\mathrm{a}}$ \\
\hline 1(7)-p-Menthene-2-one & n.d. & Menthofuran & n.d. \\
\hline 1,8 -Cineole & $\mathrm{X}$ & Menthol $^{\mathrm{a}}$ & n.d. \\
\hline 1-Cyclohexylethanone & n.d. & Menthone & n.d. \\
\hline 2,5-Diethyltetrahydofuran & n.d. & Menthyl acetate & n.d. \\
\hline 3,4,4-Trimethoxycyclohexa-2,5-dienone & n.d. & Methyl isopulegone & n.d. \\
\hline 3,5,5-Trimethylcyclohexene & n.d. & Methyl pulegone & n.d. \\
\hline 3-Hexenol & n.d. & Neoisopulegol & n.d. \\
\hline 3-Methylcyclohexanone & n.d. & Neomenthol & n.d. \\
\hline 3-Methylcyclopentanone & n.d. & Nonadecane & n.d. \\
\hline 3-Octanol & n.d. & Palmitic acid & $\mathrm{X}$ \\
\hline 3-Octanyl acetate & n.d. & p-Cymene & n.d. \\
\hline 4-Terpinyl acetate & $\mathrm{X}$ & Piperitenone & $\mathrm{X}$ \\
\hline 6,10,14-Trimethyl-pentadecanone & n.d. & Piperitinone & n.d. \\
\hline 8-Hydroxyl-p-menth-4-ene-3-one & n.d. & Piperitenol & n.d. \\
\hline Calamenene & n.d. & Piperitone & n.d. \\
\hline Camphene & $\mathrm{X}$ & Piperitone oxide & n.d. \\
\hline Camphor & n.d. & $p$-Mentha-1,8-diene-2-one & n.d. \\
\hline Carvacrol & n.d. & Pulegone & $\mathrm{X}$ \\
\hline Carvone & n.d. & Stearic acid & $\mathrm{X}$ \\
\hline Caryophyllene oxide & n.d. & Terpinen-4-ol & $\mathrm{X}$ \\
\hline Geraniol & n.d. & Thymol & n.d. \\
\hline Germacrene-D (sample 3) & $\mathrm{X}$ & Viridiflorol & $\mathrm{X}$ \\
\hline Hexanal & n.d. & $\alpha$-Humulene & $\mathrm{X}$ \\
\hline Isomenthone & n.d. & $\alpha$-Phelladrene & $\mathrm{X}$ \\
\hline Isopiperitone & n.d. & $\alpha$-Pinene & $\mathrm{X}$ \\
\hline Isopulegol & $\mathrm{X}$ & $\alpha$-Thujene & n.d. \\
\hline Isopulegone & n.d. & $\beta$-Bourbonene & n.d. \\
\hline Limonene & n.d. & $\beta$-Caryophyllene & $\mathrm{X}$ \\
\hline Linalool & n.d. & $\beta$-Ionone & n.d. \\
\hline Methyl linoleate & $\mathrm{X}$ & $\beta$-Pinene & $\mathrm{X}$ \\
\hline
\end{tabular}

a Compounds marked with an "X" were detected in samples obtained with UBE and hydrodistillation/SFE, while compounds denoted as n.d. were not detected using UBE,

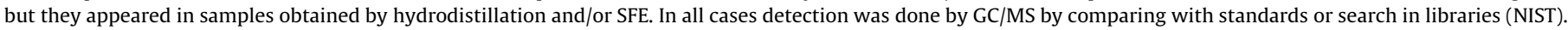


The higher content in MEFA could be due to an easier extraction of low polarity membrane lipids in contact with the organic solvent.

As far as the quantification of pulegone is concerned, a calibration curve in the range between 10 and $0.001 \mathrm{ppm}$ was previously obtained and the amount of pulegone was calculated for each set of growth conditions (solidified agar, glass beads and pots in the greenhouse) with the results shown in Table 3. Pulegone was then used as an internal standard to estimate the concentration of the other compounds identified, assuming a response factor equal to 1.0 and a linear response. The total contents in monoterpenes, sesquiterpenes and fatty acids methyl esters were also calculated on the basis of these results.

There is a clear reduction in the pulegone content from soil to in vitro conditions and this correlates well with the reduction in the essential oil production (Table 2). Also, while there is no significant difference in the essential oil content (Table 2, entries 8 and 9) and biomass production (Table 1 ) between plants grown in solidified agar or glass beads, there is a 20 -fold increase in the amount of pulegone produced that could be linked with the increase in mechanical impedance from agar to glass beads (5.59 and $110.50 \mu \mathrm{g} / \mathrm{mg}$ FW, respectively; Table 3). Likewise, it seems there is a direct correlation between the increased production of monoterpenes and the increased abiotic stress in the change from agar to glass beads (mechanical impedance) and then to greenhouse. This also holds for the total amount of terpenes. Surprisingly, a statistical difference was not found between the sesquiterepene content of trichomes between soil and agar solidified plants. Moreover, sesquiterpenes could not be detected in plants grown on glass beads. We do not have a clear explanation for this finding at present.

In vitro explants were not subjected to any other difference in the growth conditions other than the support in which they were grown. It is likely that there must be a direct correlation between the increase in pulegone biosynthesis and the mechanical impedance exerted in the roots. It has been shown previously that in the case of mint species the abiotic stressors such as UV radiation induce the production of essential oils that are rich in monoterpenes (Voirin et al., 1990). It is feasible that other abiotic environmental factors might have the same effect. This was confirmed by the almost 10 -fold increase in the pulegone content of greenhouse plants when compared to those grown under glass bead conditions ( 110.50 and $1357.35 \mu \mathrm{g} / \mathrm{mg}$ FW, respectively; Table 3 ) and the 20-fold increase when comparing agar and glass beads conditions (Table 1 ). In general, monoterpenes show the same increasing trend as pulegone on changing from in vitro to greenhouse conditions.

\subsection{Monoterpenes}

1,8-Cineole, menthone (dihydropulegone), $p$-menth-1-en-8-ol, pinocamphone, terpinen-4-ol and the sesquiterepenes germacrene $\mathrm{B}$, germacrene $\mathrm{D}$, viridiflorol and spathulenol were all detected in the agar-grown plants, but not in plants produced under other growth conditions. Camphene, $p$-menth-8-en-2-ol acetate and chrysanthenone were detected in plants from the soil and glass bead conditions, but not in agar-grown plants. $p$-Menth-8-en-3-ol (isopulegol), $p$-menth8-en-3-one, terpineol, $\gamma$-terpineol, isopulegyl acetate, carveyl acetate and phytol were detected only in plants grown in vivo on soil (Fig. 5). Despite the fact that the total amount of terpene volatiles remains more or less constant under in vitro conditions, a clear difference was found between these plants and those grown in the greenhouse.

Recently, it has been shown that at least four enzymes responsible for the biosynthesis of $p$-menthane monoterpenes are localized in the secretory cells of peltate glandular trichomes (Turner and Croteau, 2004). Also, inmunocytochemical studies show a strong connection with the secretory phase of gland development and demonstrate the importance of factors that influence trichome development as well. Consequently, all of the factors that affect trichome development will strongly influence essential oil production, a situation in good agreement with the results obtained for pulegone and for the monoterpene content. Pulegone is obtained from geranyl-PPO by cyclization and successive oxidations and dehydrogenations. Pulegone can also be transformed into less toxic monoterpene ketones and alcohols by reduction (Fig. 6). Many factors seem to influence the expression of genes involved in monoterpene biosynthesis. In particular, (+)-pulegone reductase and (+)-menthofuran synthase transcription can be upregulated by UV-B treatment, whereas (-)limonene hydroxylase transcription is down-regulated (Pino et al., 2006). An increase in (+)-pulegone has been observed in wild thyme and basil subjected to heavy goat grazing in comparison to plants growing in areas that are not exposed to these herbivores (Müller-Schwarze and Thoss, 2008), thus showing the connection between these conditions and plant defence. Other factors that have yet to be studied, including mechanical impedance, could also be involved. In this study, pulegone content increases from agar to glass bead conditions (Table 3 ), where the main difference is the increase in mechanical impedance on the roots.

Isopulegol and isopulegyl acetate were detected only in the plants grown under greenhouse conditions. These compounds are formed from pulegone through a pulegone reductase and, probably, a subsequent acetylation. According to these data, the genes that encode the pulegone reductase seem to be repressed under in vitro conditions. This is not surprising since pulegone production is enhanced as the number of developed trichomes grows in response to increased environmental stress (root impedance, water stress, UV light) and it could be a way of storing excess pulegone in a less toxic form. Interestingly, menthone (dihydropulegone), which is formed from pulegone in a reaction involving another pulegone reductase, could not be detected under greenhouse conditions, while it is present in plants grown in agar, albeit in very small amounts. The reasons for these different outcomes are as yet unknown. However, it has been shown previously that under reduced environmental pressure - e.g. in the absence of mammalian grazers - pulegone is transformed into the less toxic menthane (Pino et al., 2006). The absence of abiotic stressors such as mechanical impedance in agar conditions could lead to the same effect by enhancing (+)-pulegone reductase expression. Menthone could not be detected in the trichomes of plants grown in glass beads or in the greenhouse (Table 1 ).

Isopulegone ( $p$-menth-8-en-3-one), which is the direct biosynthetic precursor of pulegone, could only be detected in the greenhouse plants and not those grown under the other two sets of conditions. The ratio of isopulegone/pulegone in greenhouse plants is 0.0038 . The pulegone concentration exhibits a 10 -fold and $c a .20$-fold decrease in glass beads and agar, respectively. Such a decrease can cause isopulegone levels to decrease below detection limits, thus explaining why it does not appear in the other two profiles.

Menthol - another $p$-menthane that is commonly described in essential oils obtained by hydrodistillation - could not be detected in the trichome extract under any of the three growth conditions. This could indicate that production and/or storage of this compound takes place outside the trichomes - a situation supported by the fact that menthol should have been detected at least in the plants grown in the greenhouse, a fact that not happened in this study. The absence of menthol, camphor, linalool, carvone, dihydrocarvone and $\beta$-ionone (Table 5) was specifically checked by comparison with standards and it can be confirmed that none of the samples contained any of these compounds. 
RT: 3,91 - 30,08
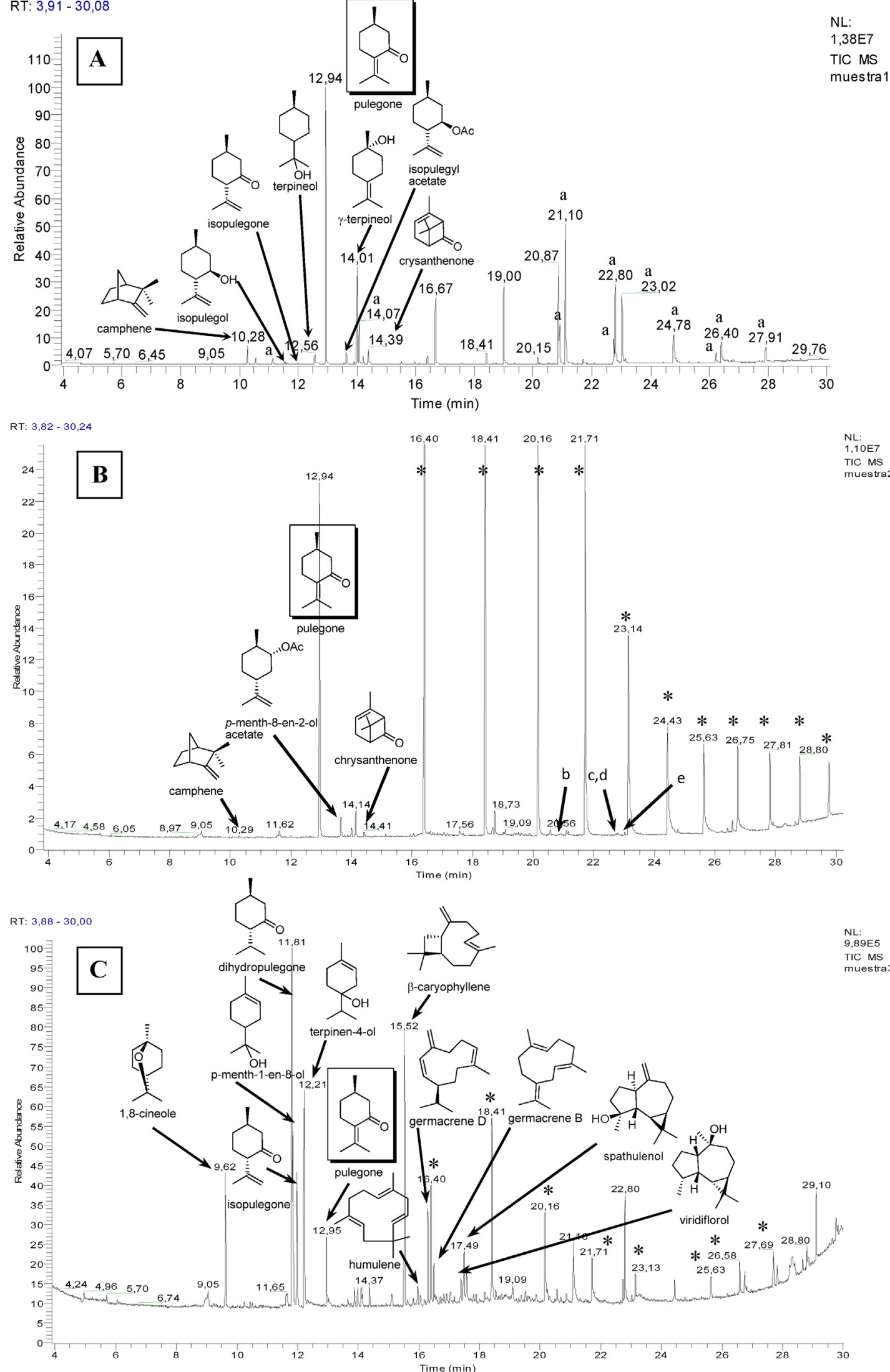

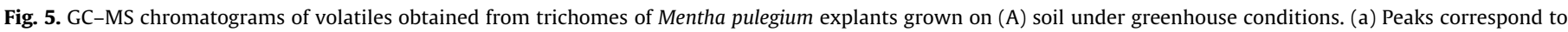

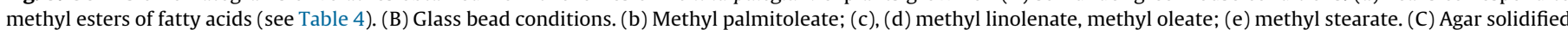
medium. *Peaks on graphs (B) and (C) correspond to silanes coming out from the column. 


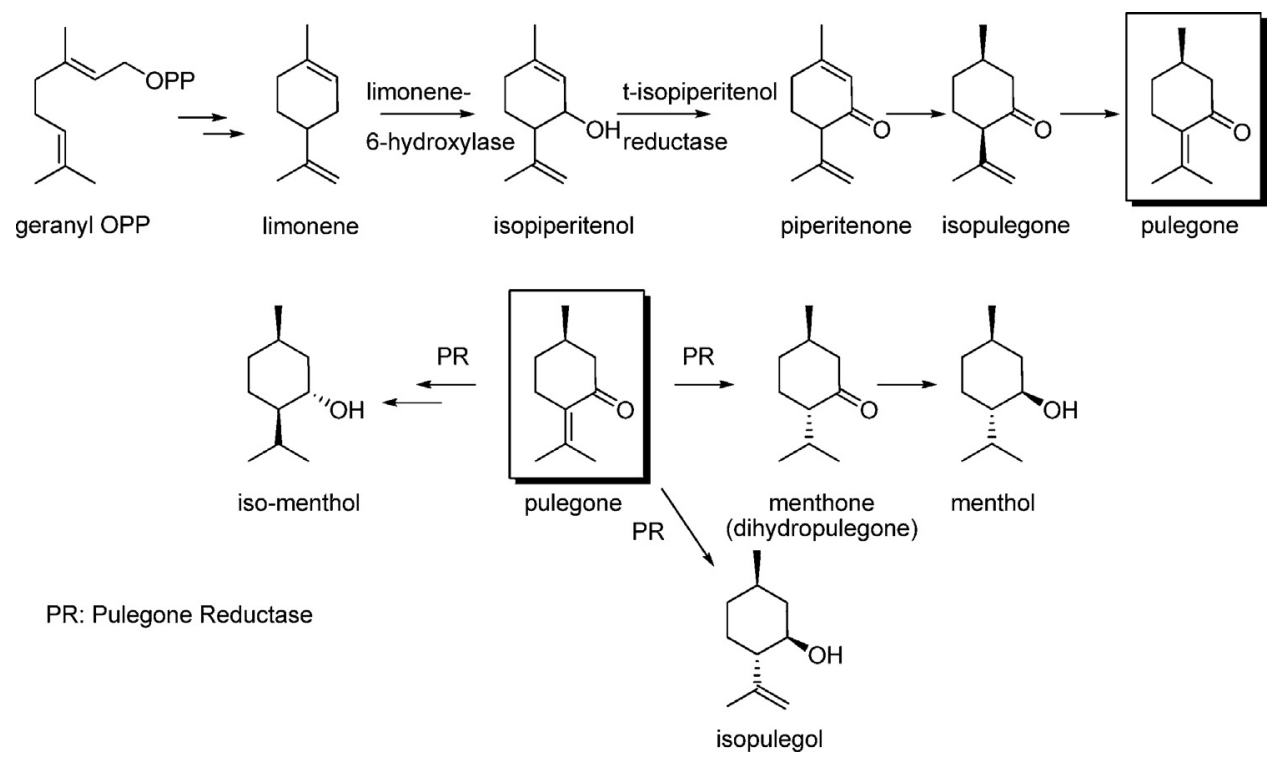

Fig. 6. Biosynthetic pathway for pulegone and its reduced forms. Adapted from Turner and Croteau (2004).

Cyclocitral (or camphone) is eluted at the same retention time as isopulegone but appears only under agar conditions. Neither cyclocitral nor camphone have been reported before in pennyroyal. 1,8-Cineole, another common allelopathic agent, appears only in plants grown on agar, but trichomes of plants grown under the other two sets of conditions do not contain 1,8-cineole.

Thus, the introduction of different degrees of mechanical impedance in explants cultured in vitro could allow the selection of growth conditions leading to plants with a high or a low content in pulegone and other volatile compounds.

\subsection{Sesquiterpenes}

The trend shown in the biosynthesis of volatile sesquiterpenes was totally unexpected, as the amounts obtained for this family under greenhouse and agar conditions were almost equal (43.36 and $31.13 \mu \mathrm{g} / \mathrm{mg} \mathrm{FW}$, respectively) but these compounds were below the limit of detection under glass bead conditions (Table 3). Such "erratic" behaviour can be explained only if the biosynthetic pathways to sesquiterpenes were knocked out under glass bead conditions, resulting in the increased production of pulegone as a response to the increased stress induced by the higher mechanical impedance on the roots. Reports of such regulation of these pathways have not been published previously, but this idea correlates well with the fact that pulegone is the main defence component of pennyroyal. At first glance, it might be proposed that the small increase in mechanical impedance from agar to glass beads redirects the carbon flow to the biosynthesis of defence monoterpenes (pulegone) but this effect is not enough to increase total volatile production. The increased mechanical impedance on the roots and its hormonal transduction to other parts of the plant correlates well with the increased monoterpene content and the increase in leaf hairs (Fig. 2), although there is no such correlation with the number of glandular trichomes. The situation equilibrated when plants were transferred to the greenhouse and grown under normal conditions. Under greenhouse conditions, all pathways resume and the synthesis of sesquiterpenes, in addition to the increased production of defence monoterpenes, reaches the same levels as for plants grown in agar.

\subsection{Esterified fatty acids}

As for the MEFA, there are also significant differences between the fatty acid profiles of plants grown under all three sets of conditions. It seems clear that there is an increase in the production of these compounds with the stress (mechanical and/or other abiotic stressors) levels: the highest content in these compounds is observed in plants grown on soil in the green house (1.83 $\mathrm{mg}, 2634.8 \mu \mathrm{g} / \mathrm{mg} \mathrm{FW}$ ), while there is a three-fold decrease when plants were grown in vitro on glass beads ( $30 \mu \mathrm{g}, 3.3 \mu \mathrm{g} / \mathrm{mg}$ FW) (Table 4). Finally, the concentration of MEFA was below the detection limits in the trichomes extract of agar-grown plants. It is noteworthy to mention that these fatty acids appear as natural methyl esters in the trichomes and they cannot be artefacts, given the extraction methodology and that no $\mathrm{MeOH}$ was used throughout all the sample processing. Essentially, eleven methyl esters could be detected in the trichomes of greenhouse plants and of these only four were detected in those of plants grown on glass beads. MEFA could not be detected in plants grown in agar. Only methyl linoleate, methyl stearate and methyl palmitate were previously reported in pennyroyal essential oil. Whether this decrease in the number and content of fatty acids could be indicative of a lower degree of development in the trichomes is an issue that has not been discussed.

From the data presented here, it seems clear that there is a correlation between the development of secondary organs and environmental stress, specifically between mechanical impedance on roots, and defence organs (i.e. trichomes and leaf hairs). Whether this connection takes place through hormone signal transduction or another mechanism is an issue for discussion.

Ultrasound extraction performed for a short period of time enabled the selective extraction of compounds stored in glandular trichomes and not those in the inner tissues, as evidenced by the appearance of the trichomes in the SEM pictures before and after treatment. Consequently, the chemical content of essential oils obtained by selective ultrasound extraction differs markedly from that obtained through hydrodistillation or supercritical fluid extraction, as previously reported in the literature.

Differences are observed between the essential profiles of explants grown in agar and glass beads, and also between these 
plants and those grown under greenhouse conditions. The main differences are found in the monoterpene profile (especially the $p$ menthane derivatives) and in the fatty acid content and profile. These differences have been correlated in this paper with an increase in the environmental stressors: firstly in the mechanical impedance exerted on the roots and, possibly, light intensity. Secondly, the possibility of a certain degree of water stress being of influence in the greenhouse plants cannot be discarded.

\section{Materials and methods}

\subsection{Plant material and in vitro culture}

All experiments were conducted with plants grown under nonsoil (in vitro) and soil (greenhouse) conditions. Original plant material was collected from M. pulegium L. growing in natural populations of North Morocco. The in vitro plants were induced from nodal explants of $M$. pulegium L. cultivated in MS (Murashige \& Skoog) medium supplemented with $0.5 \mathrm{mg} / \mathrm{L}$ BAP (6-benzylaminopurine), $30 \mathrm{mg} / \mathrm{L}$ sucrose and $7 \%$ agar (Murashige and Skoog, 1962). Micro-shoots obtained from cultured nodal explants were multiplied by sub-culturing after 3 weeks in fresh MS medium and used as explants for subsequent experiments. The methodology used herein is similar to that utilized in analogous experiments for in vitro micro-propagation from nodal explants (Ugandhar et al., 2012; Sharifi et al., 2012; Islam et al., 2010).

In experiments aimed at assessing the physical parameters that influence biomass production and secondary metabolite accumulation, micro-shoots of $4 \mathrm{~cm}$ length were planted in Plant Growth Regulator (PGR)-free fresh MS medium using two different support conditions: agar and liquid medium in a glass bead $(\varnothing 5 \mathrm{~mm})$ matrix. The cultures were multiplied and maintained by subculturing in MS medium supplemented with $30 \mathrm{mg} / \mathrm{L}$ sucrose for at least one year. All cultures were established by planting of explants and micro-shoots in a conical flask containing $10 \mathrm{ml}$ MS medium and samples were incubated $16 \mathrm{~h} / 8 \mathrm{~h}$ day/light photoperiod ( $40 \mu \mathrm{mol} \mathrm{m} \mathrm{m}^{-2} \mathrm{~s}^{-1}$ of light intensity), $24^{\circ} \mathrm{C} \pm 1$ day and night temperature. Thereafter, plants were split in two groups. On group of rooted in vitro plants were transferred to pots (one plant per pot) containing commercial potting mix in a green house in order to achieve acclimatization under natural daylight and temperature conditions and grown during 40 days prior to achieve any analysis. The other was kept in vitro under the same conditions. Plants grown on soil under greenhouse conditions were watered regularly (twice a week) to minimize the impact of water stress. The amount of water per pot was always the same for all pots and treatments.

Under non-soil conditions the morphological, histochemical and physiological parameters of rooted in vitro plants were measured after 4 weeks of subculture (time needed for total root induction). In the case of plants grown in soil, measurements of the different parameters were started one month after transplanting the plantlets to the pots. Analysis of plants grown under soil and non-soil conditions was carried out within the same period of time to assure comparativeness between the different sets of results.

\subsection{Determination of root weight, growth ratio and other physiological parameters}

The growth of shoots (biomass and growth index) was measured as described by Catapan et al. (2002). The micro-shoots were separated from the medium, blotted and weighed. The growth index (GI) was measured as the ratio of fresh weight of harvested shoots (HsFw) minus the fresh weight of planted shoots (PsFw) divided by the fresh weight of planted shoots (Eq. (1))
(Bauer et al., 2004).

$\mathrm{GI}=\frac{\mathrm{HsFw}-\mathrm{PsFw}}{\text { PsFw }}$

To determine leaf water content (LWC), fresh and lyophilized fully expanded leaves of micro-propagated and acclimatized plants of M. pulegium L. were weighed to determine the fresh (LFW) and dry (LDW) weights, respectively. Leaf water content (LWC) was recorded as the difference relative to the total FW (Cappelletti, 1954) according to Eq. (2):

$\mathrm{LWC}=\left[\frac{\mathrm{LFW}-\mathrm{LDW}}{\mathrm{LFW}}\right] \times 100$

The number of lateral roots per shoot and the number of shoots per plant were recorded from 10 in vitro plants. The remaining plants were used for the analysis of allelochemicals. All experiments were repeated at least twice.

Leaf chlorophyll (chl $a$ and chl $b$ ) content was measured by acetone extraction and spectrophotometry (Spectronic 1001, Milton Roy Co., Rochester, NY) on young leaves based on the method described by Arnon (Arnon, 1949). The contents of chl $a$, chl $b$ and chl $(a+b)$ were calculated from the light absorption readings (E), at 645 and $663 \mathrm{~nm}$ (Eq. (3)-(5)). The results of chlorophyll estimates are means of three replicates and are expressed as mg per $\mathrm{mg}$ of fresh weight of the tissue.

$\operatorname{chl}(a+b)=8.02 E_{663}+20.20 E_{645}$

chl $a=12.7 E_{663}-2.69 E_{645}$

$\operatorname{chl} b=22.9 E_{645}-4.68 E_{663}$

\subsection{Physiological status and growth kinetics}

Three parameters were used to provide clues about the biokinetics under non-soil and soil conditions: leaf area expansion, nodal growth (Gnd) and relative growth rate (RGR).

The relative growth rate (RGR) $\left(\mathrm{g} \mathrm{day}^{-1}\right.$ per plant) was determined from the nodal growth (Gnd) (nodal number day ${ }^{-1}$ per shoot) and the leaf area expansion using 10 plants from each treatment (agar, glass beads and soil). Measurements were started 30 days and 40 days, respectively, after transplanting to the pots (when active growth had started) and RGR was obtained from Eq. (6) (Gardner et al., 1985):

$\mathrm{RGR}=\frac{\text { Planted (final weight) }- \text { Planted (initial weight) }}{\text { Number of days }}$

\subsection{Morphological study of defence organs. Leaf hairs and trichomes: morphology and density}

Fifty leaves of uniform size from in vitro plants grown in glass beads and agar matrix and from plants grown in pots under greenhouse conditions were collected for electron microscopy scanning studies (SEM) (Quanta 200 scanning electron microscope). Leaves were dried overnight under vacuum. Coating with gold or gold/palladium alloy was not necessary in view of the results obtained in the initial experiments. Micrographs were recorded at $100 \times, 200 \times, 500 \times$ and $1000 \times$ magnification. The density of leaf secretory structures (i.e. capitate and peltate trichomes and simple hairs) was recorded on abaxial and adaxial sides of the leaf. Differences were not found between the samples and the results quoted are means of all experiments.

The density of secretory structures was estimated by counting them over a fixed area selected randomly on the micrograph. Three areas were counted per leaf and results are given based on the 
results of ten different leaves per culture type (agar and glass bead explants, and pots in the greenhouse). A comparative study of the leaf secretory structures was also performed before and after extraction with dichloromethane (DCM) using ultrasound under the same conditions.

\subsection{Statistics}

The data for all parameters were subjected to one way ANOVA using the statistical package available from SPSS.

\subsection{Histochemical studies. Essential oil extraction}

Fifty fresh leaves were sampled from $M$. pulegium L. grown under the three different sets of growth conditions. Leaves from each set of culture conditions were weighed and extracted for $1 \mathrm{~min}$ in $10 \mathrm{~mL}$ of DCM in separate sealed glass sample vials using ultrasound. The solvent was evaporated under vacuum in a cold bath (water with ice, $0-5{ }^{\circ} \mathrm{C}$ ) in order to avoid loss of essential oils. Samples were then redissolved in $1 \mathrm{~mL}$ of acetone for GC-MS analysis and kept sealed in the same vial at $-30{ }^{\circ} \mathrm{C}$ prior to use.

\subsection{Gas chromatography-mass spectrometry of essential oils}

GC volatile analyses were performed using a Varian CP-3800 gas chromatograph equipped with a flame ionization detector (FID) and an electronic pressure injector Model 1177 (Varian CP-8400 Autosampler) coupled with a Varian Saturn 2200 mass spectrometer with electron impact ionization (70 eV). A sample volume of $1 \mu \mathrm{L}$ was injected in split/splitless mode during $0.1 \mathrm{~min}$ (split ratio of 1:9) on a DB1 column ( $30 \mathrm{~m} \times 0.25 \mathrm{~mm}, 0.25 \mu \mathrm{m}$ film thickness) using helium as carrier gas with a flow rate of $1.6 \mathrm{~mL} / \mathrm{min}$. The analyses were performed using the following temperature programme: oven temperature isotherm at $40{ }^{\circ} \mathrm{C}$ for $3 \mathrm{~min}$, from 40 to $300{ }^{\circ} \mathrm{C}$ at a rate of $10^{\circ} \mathrm{C} \mathrm{min}{ }^{-1}$, and isotherm at $300{ }^{\circ} \mathrm{C}$ for $5 \mathrm{~min}$. The injector and detector were held, respectively, at $50{ }^{\circ} \mathrm{C}$ and $250{ }^{\circ} \mathrm{C}$. Quantitative data were obtained from the electronic integration of peak areas. The scan time and mass range in the mass spectrometer were $1 \mathrm{~s}$ and 40 $300 \mathrm{~m} / \mathrm{z}$, respectively.

Pulegone was identified by comparison of its retention time (r.t. $=12.94 \mathrm{~min}$ ) with that of the standard sample run under the same conditions and by matching its recorded mass with that stored in the NIST'98 library. Standards of $\beta$-pinene, linalool, camphor, isopulegol, menthol, dihydrocarvone, carvone, $\beta$-caryophyllene and all methylated fatty acids (MEFA) were also used to identify compounds by their retention times (r.t.) and mass spectra (Tables 3 and 4 ). The remaining compounds were tentatively identified by matching their mass spectra with those stored in the same library and by comparison with the profiles published in the literature from previous studies on $M$. pulegium L. The concentrations of identified compounds were estimated by reference to that of pulegone, which was used as the internal standard, within the same chromatogram. Each sample measurement was repeated three times.

\subsection{Calibration curves for pulegone}

A calibration curve was run with a standard sample (SigmaAldrich, Co.) at concentrations of 10, 1, 0.1, 0.01 and $0.001 \mathrm{ppm}$. Each concentration was analyzed three times and average areas obtained by integration of chromatogram peaks (FID) were correlated with the concentration $\left(r^{2}=0.9968\right.$ on FID).

\section{Acknowledgements}

K.H.B.Z. acknowledges financial support from Ministerio de Educación y Ciencia (BOE 12/1/2007 grant A/7251/06). This research was financed by the Ministerio de Innovación, Ciencia e Tecnología (MICYT), project \# AGL C009-08864(AGR) and Junta de Andalucía (Consejería de Innovación, Ciencia y Empresa, project \# P07-FGM-0303). The authors also wish to thank Dr. J.G. Romagni (Bucknell University, PN) for her useful comments on the manuscript.

\section{References}

Aghel, N., Yamini, Y., Hadjiakhoondi, A., Pourmortazavi, S.M., 2004. Supercritical carbon dioxide extraction of Mentha pulegium L. essential oil. Talanta 62, 407411.

Aitken-Christie, J., Kozai, T., Ann, M., Smith, L., 1995. Automation and environmental control in plant tissue culture. Plant Tissue Cult. 1-18.

Andre, C.M., Schafleitner, R., Legay, S., Lefevre, I., Aliaga, C.A.A., Nomberto, G., Hoffmann, L., Hausman, J.-F., Larondelle, Y., Evers, D., 2009. Gene expression changes related to the production of phenolic compounds in potato tubers grown under drought stress. Phytochemistry 70, 1107-1116.

Arnon, D.I., 1949. Copper enzymes in isolated chloroplasts. Polyphenoloxidase in Beta vulgaris. Plant Physiol. 24, 1-15.

Ascensão, L., Marques, N., Pais, M.S., 1995. Glandular trichomes on vegetative and reproductive organs of Leonotis leonurus (Lamiaceae). Ann. Bot. 75, 619-626.

Banchio, E., Valladares, G., Zygadio, J., Bogino, P.C., Rinaldo, L.V., Giordano, W., 2007. Changes in the composition of essential oils and volatile emmisions of Minthostachys mollis, induced by leaf punctures of Liriomyza huidobrensis. Biochem. Syst. Ecol. 35, 68-74.

Bauer, N., Leljalk-Levanic, D., Jelaska, S., 2004. Rosmarinic acid synthesis in transformed callus culture of Coleous blumei Benth. Z. Naturforsch. 59c, 554-560.

Bengoung, A.G., McKenzie, B.M., Hallet, P.D., Valentine, T.A., 2011. Root elongation, water stress, and mechanical impedance: a review of limiting stresses and beneficial root tip traits. J. Exp. Bot. 82, 59-68.

Braam, J., 2005. In touch: plant responses to mechanical stimuli. New Phytol. 165, 373-438.

Canter, P.H., Thomas, H., Ernst, E., 2005. Bringing medicinal plants into cultivation: opportunities and challenges for biotechnology. Trends Biotech. 23, 4.

Cappelletti, C., 1954. Water content in plants and equations used to determine it. Ann. Bot. Roma 24, 408-430.

Catapan, E., Luis, M., da Silva, B., Netto Moreno, F., Viana, A.M., 2002. Micropropagation, callus and root culture of Phyllantus urinaria (Euphorbiaceae). Plant Cell Tissue Organ Cult. 70, 301-309.

Conconi, A., Smerdon, M.J., Howe, G.A., Ryan, C.A., 1996. The octadecanoid signalling pathway in plants mediates a response to ultraviolet radiation. Nature 383 826-882.

Croteau, R., Kutchan, T.M., Lews, N.G., 2000. Natural products (secondary metabolites). In: Buchanan, B.B., Gruissem, W., Jones, R.L. (Eds.), Biochemistry and Molecular Biology of Plants. American Society of Plant Physiologists, Rockville, pp. 1250-1318.

Diaz-Maroto, M.C., Castillo, N., Castro-Vazquez, L., Gonzalez-Vinas, M.A., PerezCoello, M.S., 2007. Volatile composition and olfactory profile of pennyroyal (Mentha pulegium L.) plants. Flavour Fragr. J. 22, 114-118.

Duffey, S.S., 1986. Plant glandular trichomes: their partial role in defense against insects. In: Juniper, B.E., Southwood, T.R.E. (Eds.), Insects and the Plant Surface. Edward Arnold, London, pp. 151-172.

Eisner, T., McCormick, K.D., Sakaino, M., Eisner, M., Smedley, S.R., Aneshansley, D.J., Deyrup, M., Myers, R.L., Meinwald, J., 1990. Chemical defense of a rare mint plant. Chemoecology 1, 30-37.

Etienne, H., Berthouly, M., 2002. Temporary immersion systems in plant micropropagation. Plant Cell Tissue Organ Cult. 69, 215-231.

Fasano, J.M., Massa, G.D., Gilroy, S., 2002. Ionic signaling in plant responses to gravity and touch. J. Plant Growth Reg. 21, 71-88.

Gang, D.R., Simon, J., Lewinsohn, E., Pichersky, E.J., 2002. Peltate glandular trichomes of Ocimum basilicum L. (sweet basil) contain high levels of enzymes involved in the biosynthesis of phenylpropenes. Herbs Spices Med. Plants 9, 189-195.

Gardner, F.P., Pearce, R.B., Mitchell, R., 1985. Physiology of Crop Plants. Iowa State University Press, Ames, pp. 187-208.

Goel, M.K., Kukreja, A.K., Khanuja, S.P.S., 2007. Cost effective approaches for in vitro mass propagation of Rauwolfia serpentina Benth. ex Kurz. Asian J. Plant Sci. 6, 961-975.

Goeschl, J.D., Rappaport, L., Pratt, H.K., 1996. Ethylene as a factor regulating the growth of pea epicotyls subjected to mechanical stress. Plant Physiol. 41, 877884

Herms, D.A., 1999. Physiological and abiotic determinants of competitive ability and herbivore resistance. Phyton 39, 53-64.

Herms, D.A., Mattson, W.J., 1992. The dilemma of plants: to grow or defend. Q. Rev. Biol. 67, 283-335.

Islam, S., Zahan, M., Akter, S., Banu, T.A., Habib, A., Khan, S., Jahan, M.A.A., 2010. Mass propagation of Feronia limonia L. through tissue culture. Bangladesh J. Sci. Ind. Res. 45, 75-78.

Karousou, R., Bosabalidis, A.M., Kokkini, S., 1992. Sideritis syriaca ssp. syriaca: glandular trichome structure and development in relation to systematics. Nordic J. Bot. 12, 31-37.

Karray-Bouraoui, N., Rahbi, M., Nefatti, M., Baldan, B., Ranieri, A., Marzouk, B., Lachaâl, M., Smaoui, A., 2009. Salt effect on yield and composition of shoot 
essential oil and trichome morphology and density on leaves of Mentha pulegium. Indian Crops Prod. 30, 338-343.

Kays, S.J., Crocker, T.F., Worley, R.E., 1975. Concentration dependencies of ethylene on shuck dehiscence and fruit and leaf abscission of Carya illinoensis. J. Agric. Food Chem. 23, 1116-1118.

Kenneth, M., Jerzy, N., 1990. Glass beads as a solid matrix in in vitro study of the role of polyamines in cold hardiness of white clover. Plant Cell Tissue Organ Cult. 22, 113-117.

Khojasteh, S.C., Hartley, D.P., Ford, K.A., Uppal, H., Oishi, S., Nelson, S.D., 2012. Characterization of rat liver proteins adducted by reactive metabolites of menthofuran. Chem. Res. Toxicol. 25, 2301-2309.

Liu, Ch.-Z., Gao, M., Guo, B., 2008. Plant regeneration of Erigeron breviscapus (vant.) Hand. Mazz. and its chromatographic fingerprint analysis for quality controls. Plant Cell Rep. 27, 39-45.

Liu, H.J., Xu, Y., Liu, Y.J., Liu, C.Z., 2006. Plant regeneration from leaf explants of Rhodiola fastigiata. In Vitro Cell Dev. Biol. Plant. 4, 345-347.

Liu, Z.L., Chu, S.S., Jiang, G.H., 2011. Toxicity of Schizonepeta multifida essential oil and its constituent compounds towards two grain storage insects. J. Sci. Food Agric. 91, 905-908.

Maruyama, K., Takeda, M., Kidokoro, S., Yamada, K., Sakuma, Y., Urano, K., Fujita, M., Yoshiwara, K., Matsukura, S., Morishita, Y., Sasaki, R., Suzuki, H., Saito, K., Shibata, D., Shinozaki, K., Yamaguchi-Shinozaki, K., 2009. Metabolic pathways involved in cold acclimation identified by integrated analysis of metabolites and transcripts regulated by DREB1A and DREB2A. Plant Physiol. 150, 1972 1980.

McClanahan, R.H., Thomassen, D., Slattery, J.T., Nelson, S.D., 1989. Metabolic activation of (R)-(+)-pulegone to a reactive enonal that covalently binds to mouse liver proteins. Chem. Res. Toxicol. 2, 349-355.

Müller-Schwarze, D., Thoss, V., 2008. Defense on the rocks: low monoterpenoid levels in plants of pillars without mammalian herbivores. J. Chem. Ecol. 34, 1377-1381.

Murashige, T., Skoog, F., 1962. A revised medium for rapid growth and bioassay with tobacco tissue culture. Physiol. Plant. 15, 473-497.

Murch, S.J., Krishna Raj, S., Saxena, P.K., 2000. Phytopharmaceuticals: mass production, standard, and conservation. Sci. Rev. Altern. Med. 4, 39-43.

Nasir Khan, M., Siddiqui, M.H., Mohammad, F., Naeem, M., Khan, M., Masroor, A. 2010. Calcium chloride and gibberellic acid protect linseed (Linum usitatissimum L.) from $\mathrm{NaCl}$ stress by inducing antioxidative defence system and osmoprotectant accumulation. Acta Physiol. Plant. 32, 121-132.

Okamoto, T., Tsurumi, S., Shibasaki, K., Obana, Y., Takaji, H., Oono, Y., Rahman, A., 2008. Genetic dissection of hormonal responses in the roots of Arabidopsis grown under continuous mechanical impedance. Plant Physiol. 146, 16511662.

Pino, L., Maffei, M., Castaldo, D., 2006. Preliminary studies on the effects of UV-B mediation on the expression of some genes involved in the biosynthesis of monoterpenes in Mentha x piperita L. Derivati Agrumari 76, 5-9.

Pirzad, A., Shakiba, M.R., Zehtab-Salmasi, S., Mohammadi, S.A., Darvishzadeh, R., Samadi, A., 2011. Effect of water stress on leaf relative water content, chlorophyll, proline and soluble carbohydrates in Matricaria chamomilla L. J. Med. Plant Res. 5, 2483-2488.
Prakash, L., Majid, D., Acram, T., 2002. Production of four commercially cultivated Echinacea species by different methods of in vitro regeneration. J. Hort. Sci. Biotech. 77, 158-163.

Rathore, J.S., Rathore, V., Shekhawat, N.S., Singh, R.P., Liler, G., Phulwaria, M., Dagla, H.R., 2004. Micropropagation of woody plants. Plant Biotech. Mol. Markers 195205.

Sahoo, Y., Chand, P.K., 1998. Micropropagation of Vitex negundo L., a woody aromatic medicinal shrub, through high-frequency axillary shoot proliferation. Plant Cel Rep. 18, 301-307.

Sampson, B.J., Tabanca, N., Kirimer, N., Demirci, B., Baser, K.H., Khan, I.A., Spiers, J.M., Wedge, D.E., 2005. Insecticidal activity of 23 essential oils and their major compounds against adult Lipaphis pseudobrassicae (Davis) (Aphididae: Homoptera). Pest Manag. Sci. 61, 1122-1128.

Sharifi, S., Nejad Sattari, T., Zebarjadi, A., Majd, A., Gashempour, H.R., 2012. Enhanced callus induction and high-efficiency plant regeneration in Tribulus terrestris L., an important medicinal plant. J. Med. Plant Res. 6, 4401-44012.

Sharma, H.C., Crouch, J.H., Sharma, K.K., Seetharrama, N.M., Hash, C.T., 2002. Application of biotechnology for crop improvement: prospects and constraints. Plant Sci. 163, 381-395.

Sistrunk, M.L., Antosiewicz, D.M., Purugganan, M.M., Braam, J., 1994. Arabidopsis TCH3 encodes a novel $\mathrm{Ca}^{2+}$ binding protein and shows environmentally induced and tissue-specific regulation. Plant Cell 6, 1553-1565.

Teixeira, B., Marques, A., Ramos, C., Batista, I.,Serrano, C., Matos, O.,Neng, N.R., Nogueira, J.M.F., Saraiva, J.A., Nunes, M.L., 2013. European pennyroyal (Mentha pulegium) from Portugal: chemical composition of essential oil and antioxidant and antimicrobial properties of extracts and essential oil. Ind. Crops Prod. 36, 81-87.

Thomassen, D., Slattery, J.T., Nelson, S.D., 1990. Menthofuran-dependent and independent aspects of pulegone hepatotoxicity: roles of glutathione. J. Pharmacol. Exp. Ther. 253, 567-572.

Tumova, L., Rimakova, J., Tuma, J., Dusek, J., 2007. Glycyrrhiza glabra culture in vitrosaponin production. Cereal Res. Commun. 35, 1233-1236.

Turner, G.W., Croteau, R., 2004. Organization of monoterpene biosynthesis in Mentha. Immunocytochemical localizations of geranyl diphosphate synthase, limonene-6-hydroxylase, isopiperitenol dehydrogenase, and pulegone reductase. Plant Physiol. 136, 4215-4227.

Ugandhar, T., Venkateshvarlu, M., Sammallah, D., Jahan Mogan Reddy, K., 2012. Rapid in vitro micro propagation of chick pea (Cicer arietinum L.) from shoot tip and cotyledonary node explants. J. Biothech. Biomat. 2, 1000148/1-1000148/6.

Vinocur, B., Carmi, T., Altman, A., Ziv, M., 2000. Enhanced bud regeneration in aspen (Populus tremula L.) roots cultured in liquid media. Plant Cell Rep. 19, 1146-1154.

Voirin, B., Brun, N., Bayet, C., 1990. Effects of daylength on the monoterpene concentration of Mentha x piperita. Phytochemistry 29, 749-755.

Wagner, G.J., 1991. Secreting glandular trichomes: more than just hairs. Plant Physiol. 96, 675-679

Xu, W., Puragganan, M.M., Polisensky, D.H., Antosiewicz, D.M., Fry, S.C., Braam, J., 1995. Arabidopsis TCH4, regulated by hormone and the environment, encodes a xyloglucan endotransglycosylase. Plant Cell 7, 1555-1567.

Yoshimatsu, K., 2008. Tissue culture of medicinal plants: micropropagation, transformation and production of useful secondary metabolites. Stud. Nat. Prod. Chem. 34, 647-752. 